\title{
Sistema de Coleta de Dados para Avaliação da Aprendizagem em Ambientes Virtuais de Aprendizagem Tridimensionais
}

\author{
Eunice P. dos Santos Nunes ${ }^{1,2,3}$, Rafael Luiz Testa ${ }^{3}$, Fátima L. S. Nunes ${ }^{1,3}$ \\ ${ }^{1}$ Interlab - Laboratório de Tecnologias Interativas - Escola Politécnica/USP
}

Av. Prof. Luciano Gualberto, no 380 - 05508-010 - São Paulo - fone (11) 3091-5282

${ }^{2}$ Instituto de Computação - Universidade Federal de Mato Grosso (UFMT)

Av. Fernando C. da Costa, no 2367 - 78060-900 - Cuiabá-MT - fone: (65) 3615-8791

${ }^{3}$ LApIS - Laboratório de Aplicações de Informática em Saúde

Programa de Pós-graduação em Sistemas de Informação - Escola de Artes, Ciências de Humanidades/USP

Av. Arlindo Béttio, 1000 - Ermelino Matarazzo - São Paulo - CEP: 03828-000

\{eunice.poli, rafael.testa, fatima.nunes\}@usp.br

\begin{abstract}
Virtual Reality and Augmented Reality technologies favor the development of more interactive and immersive human-computer interfaces. These interfaces have increased the interest of researchers to define ThreeDimensional (3D) Virtual Learning Environments (VLEs) in different contexts, but collect data to assess the learning by using these environments is still a challenge. This paper presents the Learning Assessment System (LASp) which includes instruments to diagnostic assessment and records the user interactions in the virtual environment. By analyzing the collected data the evaluator can measure how and how much 3D VLEs collaborate with the process of knowledge acquisition. The results showed that the LASp is efficient to facilitate the data collection and allows monitoring interactions that occur automatically, without interfering in the application source code.
\end{abstract}

Resumo. As tecnologias de Realidade Virtual e Realidade Aumentada favorecem o desenvolvimento de interfaces humano-computador mais interativas e imersivas. Tais interfaces têm aumentado o interesse dos pesquisadores em conceber Ambientes Virtuais de Aprendizagem Tridimensionais (AVAs 3D) em diferentes contextos, mas coletar dados para avaliar o aprendizado usando esses ambientes ainda é um desafio. Este artigo apresenta o Sistema de Avaliação de Aprendizagem (SAAp), que inclui instrumentos para avaliação diagnóstica e registro das interações do usuário no ambiente virtual. Ao analisar os dados coletados, o avaliador pode medir como e quanto os AVAs $3 D$ colaboram com o processo de aquisição de conhecimento. Os resultados mostraram que o SAAp é eficiente para facilitar a coleta de dados e permite monitorar as interações que ocorrem automaticamente, sem interferir no código fonte da aplicação.

\section{Introdução}

As tecnologias de Realidade Virtual (RV) e Realidade Aumentada (RA) estão cada vez mais presentes no cotidiano das pessoas, principalmente na área de entretenimento. Os 
recursos computacionais proporcionados por tais tecnologias favorecem $o$ desenvolvimento de interfaces humano-computador mais envolventes, interativas e imersivas, o que têm despertado o interesse de pesquisadores em conceber Ambientes Virtuais de Aprendizagem Tridimensionais (AVAs 3D) cada vez mais sofisticados.

A exploração de AVAs 3D com propósito de ensino e aprendizagem, a partir de cenários de situações reais, vem sendo uma prática muito comum em diversos campos de conhecimento, como instrumento de apoio pedagógico, tanto no ensino presencial quanto a distância. Uma das características fundamentais dos AVAs que fazem uso dos recursos de RV e RA é o fato de se caracterizarem como ambientes virtuais dinâmicos, ou seja, o ambiente modifica-se em tempo real à medida que os usuários interagem com ele, sendo que a interação pode ocorrer em menor ou maior grau, dependendo da interface adotada [Schlemmer e Backes 2008].

Aliada a esta característica, muitos pesquisadores têm defendido a hipótese de que Ambientes Virtuais Tridimensionais (AVs 3D) são tendências na área educacional, pois permitem extrapolar os limites espaço-temporais, possibilitando, por exemplo, a visitação "virtual" a lugares que são muito pequenos para se explorar na vida real, ou lugares de extensão muito grande que possam ser visualizados como um todo, favorecendo o aprendizado experimental, simulações de treinamento em cenários complexos e contextos educacionais com maior realismo [Sherman e Craig 2003, Vendruscolo et al. 2005, Valente e Mattar 2007].

Uma Revisão Sistemática (RS) realizada por Nunes et al. (2010, 2012) na literatura especializada, verificou que existem diversas iniciativas de pesquisa em relação à concepção de AVAs 3D. Contudo, dos trabalhos incluídos na seleção final da RS, observou-se que 55\% deles utilizam algum tipo de instrumento de avaliação em relação à aprendizagem, motivação ou engajamento no ambiente, porém específicos para cada aplicação em estudo. Complementarmente, foi verificado que $45 \%$ dos trabalhos não apresentam instrumentos de avaliação. Mikropoulos e Natsis (2011) apresenta uma revisão crítica de dez anos (1999-2009) de pesquisa empírica sobre aplicações educacionais neste contexto, comprovando que há diversos estudos propondo AVAs 3D, mas pouco pode ser concluído em relação à retenção dos conhecimentos adquiridos a partir do uso desses ambientes.

A RS realizada por Nunes et al. $(2010,2012)$ indica que os instrumentos de avaliação são, em geral, provas objetivas, questionários aplicados nas fase de pré e/ou pós-treinamento, tarefas pós-teste e teste de resolução de problemas pós-treinamento. Esses instrumentos são específicos para cada aplicação. No entanto, além de aplicar instrumentos de avaliação acerca do conteúdo abordado no contexto em estudo, se faz necessário verificar o comportamento dos aprendizes nos AVAs 3D durante o processo de aprendizagem, como forma de investigar se tais ambientes favorecem a aquisição de conhecimento. Assim, uma das estratégias é medir o nível de interação do aprendiz durante a exploração no ambiente virtual, para posteriormente verificar se tais interações apresentam correlação com a evolução do aprendizado. No entanto, este monitoramento das interações pode ser trabalhoso e até mesmo inviável se executado manualmente.

O presente trabalho faz parte de um contexto maior que visa a estabelecer uma metodologia para medir a aquisição de conhecimento em AVAs 3D. O objetivo principal deste artigo é apresentar o desenvolvimento do Sistema de Avaliação da 
Aprendizagem (SAAp) que agrega Ambientes Virtuais de Aprendizagem Tridimensionais com propósito de capturar as interações dos usuários, de forma automática, durante o processo de aprendizagem. O SAAp foi desenvolvido com base no Modelo Teórico de Avaliação da Aquisição de Conhecimento proposto por Nunes et al. (2012). A Arquitetura do Sistema é apresentada na seção 2 juntamente com a implementação do módulo de monitoramento das interações que tem como diferencial não interferir no código fonte da aplicação (AVA 3D). A seção 3 apresenta os resultados da implementação e as conclusões obtidas são apresentadas na seção 4.

\section{Metodologia}

A implementação do Sistema de Avaliação da Aprendizagem teve como base o Modelo Teórico de Avaliação da Aquisição de Conhecimento (Figura 1) e sua arquitetura computacional (Figura 2), propostos por Nunes et al. (2012).

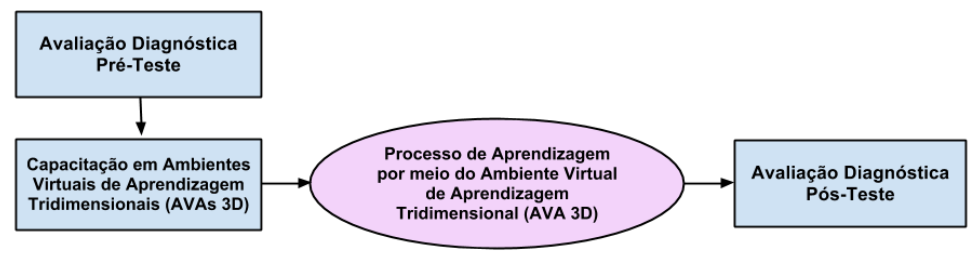

Figura 1. Modelo Teórico de Avaliação da Aquisição de Conhecimento [Nunes et al. 2012]

O Modelo Teórico de Avaliação da Aquisição de Conhecimento é baseado na perspectiva das Teorias Cognitivas, tendo em vista que busca verificar se ocorrem mudanças no comportamento dos aprendizes entre as fases de Avaliação Diagnóstica Pré-Teste e Pós-Teste. A arquitetura do sistema (Figura 2) está dividida em dois componentes principais: i) Interface do AVA 3D e ii) Sistema de Avaliação da Aprendizagem. O componente "Sistema de Avaliação da Aprendizagem" inclui os instrumentos de avaliação diagnóstica previstos. Adicionalmente, captura as interações do usuário durante a exploração no AVA 3D com propósito de coletar dados que irão nortear o avaliador a fazer julgamentos sobre a aprendizagem dos estudantes. Tal avaliação tem como base os dados das interações e os dados coletados pelos instrumentos de avaliação, oferecendo ao avaliador condições de verificar se tais ambientes estão de fato colaborando com a aquisição de conhecimento dos aprendizes.

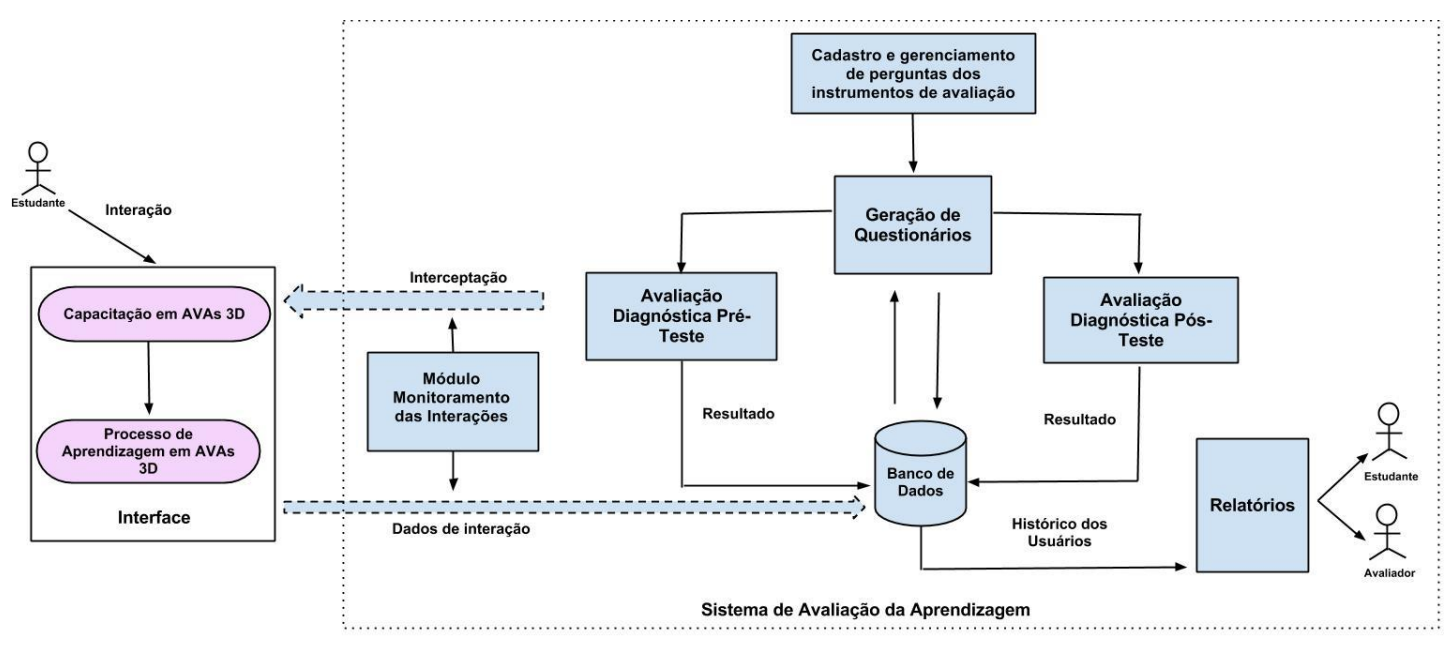

Figura 2. Arquitetura do Sistema de Avaliação da Aprendizagem [Nunes et al. 2012] 
Para implementação da arquitetura do sistema considerando todas as suas especificidades, são apresentadas na seção 2.1 as tecnologias utilizadas.

\subsection{Tecnologias Usadas}

Para viabilizar a implementação do sistema foi utilizado o paradigma de Programação Orientada a Aspectos (POA) que tem como objetivo modularizar interesses transversais que ficam misturados e distribuídos dentro do próprio código quando se utiliza apenas o paradigma de orientação a objetos. Assim, a POA permite que os módulos sejam organizados de forma independente, devido ao conceito de aspecto, permitindo que o código seja encapsulado e modularizado [Camargo e Masiero 2006]. A partir desses conceitos, foi possível desenvolver o sistema para capturar dados de interação dos usuários sem a necessidade de interferir no código fonte dos AVAs 3D.

Dessa forma, considerando as especificidades da arquitetura do sistema desenvolveu-se um módulo de registro dos dados das interações dos usuários com base na POA, por meio da extensão AspectJ [Aspectj 2013]. Para implementação do sistema optou-se pela linguagem de programação Java, por ser uma linguagem de programação que se integra diretamente com a extensão AspectJ.

O Sistema Gerenciador de Banco de Dados (SGBD) escolhido para implementação da base de dados foi o h2 Database Engine ${ }^{1}$ por ser um sistema de banco de dados embarcado, o qual permite o gerenciamento dos dados de forma integrada à aplicação e sem requerer instalações e configurações especiais.

\subsection{Monitoramento das Interações dos Usuários}

A partir das tecnologias citadas, foi definido o Módulo de Monitoramento das Interações dos Usuários e seus componentes (Figura 3). Este módulo está integrado ao Sistema de Avaliação da Aprendizagem e compartilha o mesmo banco de dados, com os seguintes componentes:

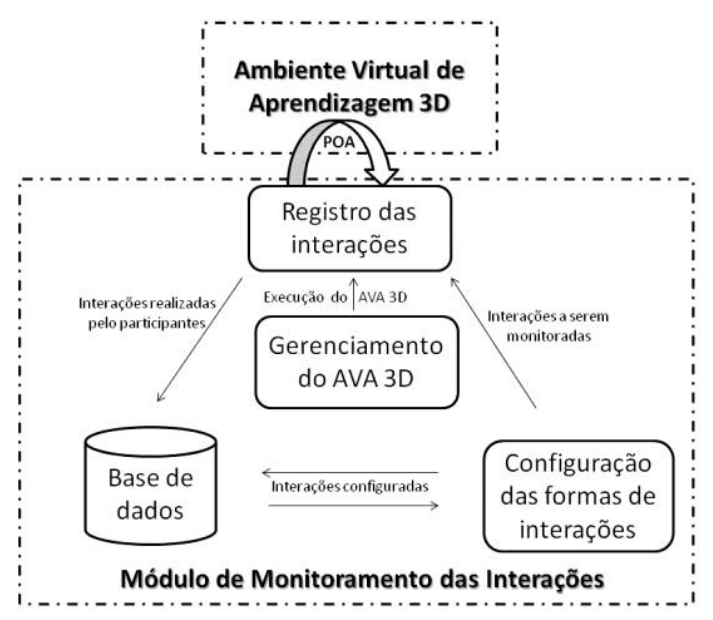

Figura 3. Módulo de Monitoramento das Interações dos Usuários

\footnotetext{
${ }^{1} \mathrm{H} 2$ Database Engine é um banco de dados de código livre escrito em Java, e pode ser encontrado em http://www.h2database.com.
} 
Registro das interações - são interceptadas as interações realizadas pelo participante durante a exploração no AVA 3D; registra-se também o tempo de cada interação e o tempo total de exploração no AVA 3D. Tais interações são registradas, somente, se forem previamente cadastradas pelo avaliador no Sistema de Avaliação da Aprendizagem. As interações são armazenadas em tempo real durante a execução da interação no próprio ambiente.

Gerenciamento do AVA 3D - tem como propósito gerenciar o cadastro, edição ou exclusão do AVA 3D (aplicação), no qual as interações são monitoradas.

Configuração das formas de interações - responsável pelo cadastro/edição/exclusão dos possíveis tipos de interações a serem monitoradas no AVA 3D, ou seja, quais os tipos de interações serão possíveis de serem capturadas quando o participante estiver explorando o ambiente e quais os eventos associados. $\mathrm{O}$ avaliador cadastra os tipos de interações a serem monitoradas, executando-as no próprio AVA 3D.

Com a integração do módulo de monitoramento das interações ao Sistema de Avaliação da Aprendizagem, pretende-se interceptar todos os eventos previamente definidos, obtendo sucesso no registro das interações dos usuários.

\subsection{Sistema de Avaliação da Aprendizagem (SAAp)}

Inicialmente, a primeira versão do sistema foi desenvolvida para ambientes desktop. Ao executar o Sistema de Avaliação da Aprendizagem, o primeiro passo é realizar o acesso do usuário no sistema de autenticação, conforme a interface ilustrada na Figura 4. A validação do sistema prevê três perfis de usuários: i) administrador; ii) avaliador e iii) participante (aluno).

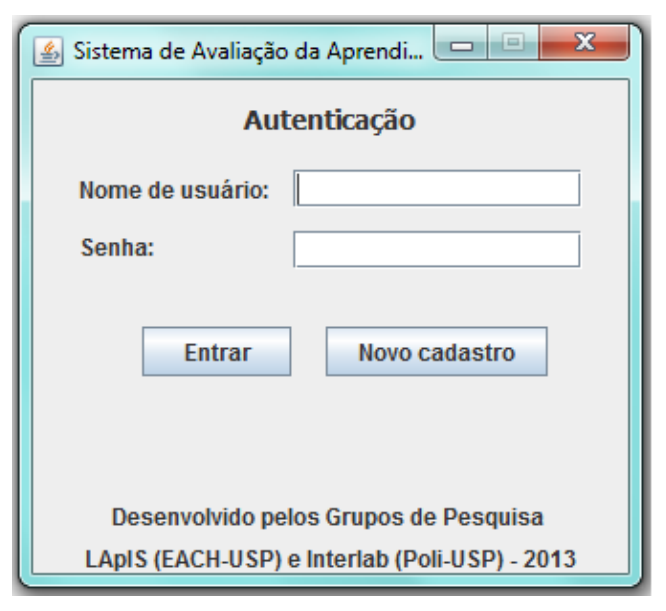

Figura 4. Interface de autenticação

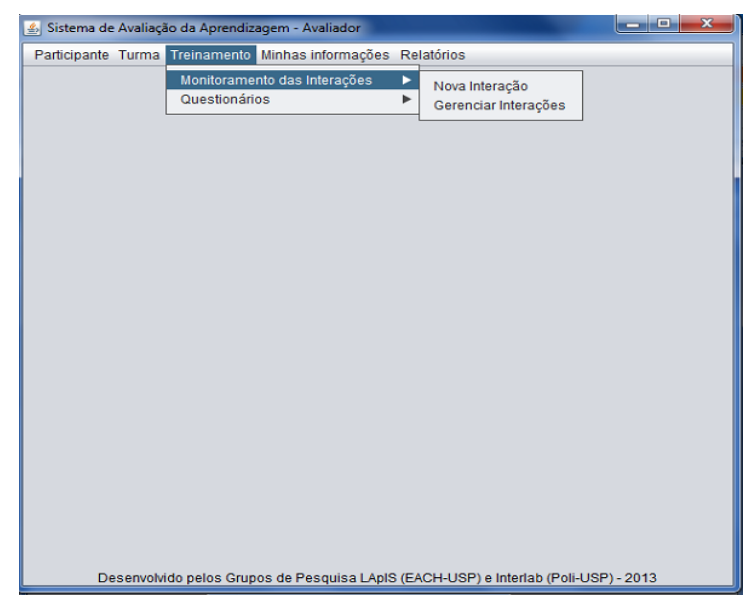

Figura 5. Interface (perfil Avaliador)

Ao acessar o sistema no perfil de avaliador, a interface ilustrada na Figura 5 é exibida ao usuário, apresentando as seguintes funcionalidades:

Turma - o avaliador cria a turma e realiza o pré-cadastro dos participantes adicionando o e-mail de cada participante na referida turma.

Participante (aluno) - permite buscar participante da turma, usando como chave de busca o nome do aluno, visando consultar e/ou alterar os dados do participante. Para tal, o participante deve ter concluído o seu cadastro por meio da interface de 
autenticação (novo cadastro), desde que este já tenha sido pré-cadastrado pelo avaliador.

Treinamento - o módulo de monitoramento das interações dos usuários está integrado a esta funcionalidade, acessando-se a opção Nova Interação. Antes de qualquer treinamento ser iniciado o avaliador deve cadastrar todas as interações possíveis, as quais ele gostaria que fossem monitoradas quando o participante realizar o treinamento. Ainda nesta funcionalidade (Treinamento), acessando a opção Questionários, é permitido ao avaliador cadastrar todas as questões a serem inseridas nas Avaliações Diagnósticas de Pré-Teste e Pós-Teste, como também cadastrar todos os questionários referentes ao processo de avaliação. Vale salientar que as questões podem ser do tipo objetivas e/ou dissertativas. As questões objetivas são corrigidas automaticamente pelo sistema conforme o gabarito cadastrado pelo avaliador. As questões dissertativas devem ter a nota atribuída pelo próprio avaliador.

Minhas Informações - permite visualizar e/ou alterar os dados do avaliador.

Relatórios - disponibiliza relatórios com os dados das interações dos usuários e os resultados obtidos por meio dos questionários aplicados no processo de avaliação da aprendizagem.

No perfil de participante (aluno), ao executar o sistema pela primeira vez, é necessário que o usuário realize um novo cadastro por meio da interface de autenticação. Após completar o cadastro, a interface ilustrada na Figura 6 é exibida ao usuário. As funcionalidades disponíveis neste perfil se referem à fase de treinamento virtual no AVA 3D, como também à capacitação em Ambientes Virtuais Tridimensionais para promover a familiaridade com tais ambientes. Neste perfil o módulo de monitoramento das interações dos usuários está integrado à funcionalidade Treinamento, o que inclui o registro das interações do usuário durante a exploração no ambiente virtual. Os questionários vinculados à avaliação da aprendizagem também são disponibilizados aos participantes do processo.

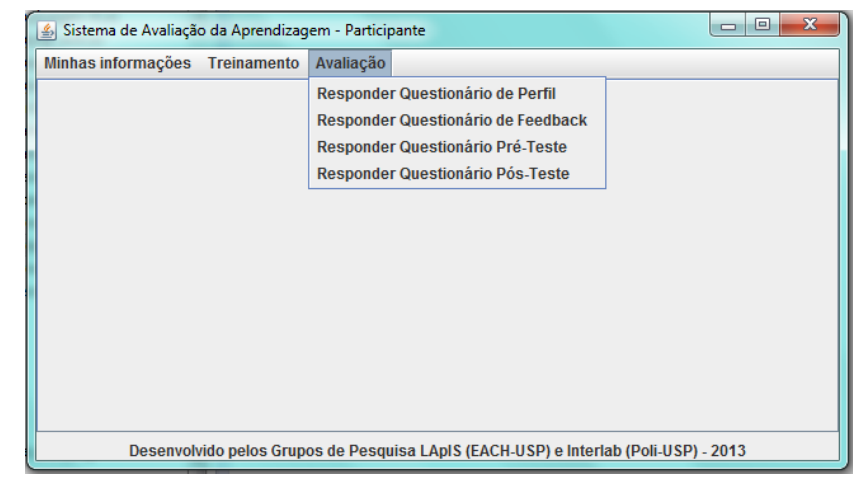

Figura 6. Interface (perfil Participante)

Com o Sistema de Avaliação da Aprendizagem em funcionamento foi possível realizar os testes do Módulo de Monitoramento das Interações dos Usuários a partir dos estudos de caso selecionados e apresentados na seção 3.

\section{Resultados e Discussões}

O SAAp torna a aplicação dos instrumentos de avaliação nas fases de Avaliação Diagnóstica Pré-Teste e Pós-Teste mais prática e rápida, pelo fato dos instrumentos estarem implementados computacionalmente. Além disso, possibilita que as interações dos usuários sejam monitoradas nos AVAs 3D sem a necessidade de alteração no 
código fonte da aplicação. A fim de comprovar o funcionamento do SAAp, foram conduzidos experimentos com dois estudos de caso: (1) Projeto VIDA (Virtual and Interactive Distance-Learning on Anatomy) [TORI et al. 2009], desenvolvido pelo Interlab/USP e pelo LApIS (Laboratório de Aplicações de Informática em Saúde) da Escola de Artes Ciências e Humanidades da USP e (2) CONSTRUFIG3D desenvolvido por um grupo de pesquisadores da Universidade Severino Sombra em Vassouras-RJ, com propósito de apoiar o ensino de Geometria Plana e Espacial [Mendes, Carvalho e Carvalho 2007].

Os primeiros testes foram realizados com o Projeto VIDA. Na versão utilizada neste estudo, o sistema VIDA apresenta as diferentes fases de um feto (3D) durante sua gestação, com propósito de identificar as etapas da evolução, período de gestação, entre outras informações de interesse dos alunos dos cursos da área de saúde. O ambiente suporta apenas a interação com dispositivos comuns, como mouse e teclado.

Na Figura 7 pode ser observada a interface do sistema VIDA agregada ao SAAp (perfil avaliador), na qual o avaliador pode interagir com o modelo tridimensional de um feto. $\mathrm{O}$ registro das interações é armazenado em tempo real durante a execução da interação no próprio ambiente, como ilustram as Figuras 7 e 8. Logo após a interação realizada, aparecerá a interface para confirmar ou refazer a interação (Figura 8). Antes de qualquer treinamento ser iniciado o avaliador deve cadastrar todas as interações possíveis, as quais ele gostaria que fossem monitoradas quando o participante realizar o treinamento no ambiente virtual.

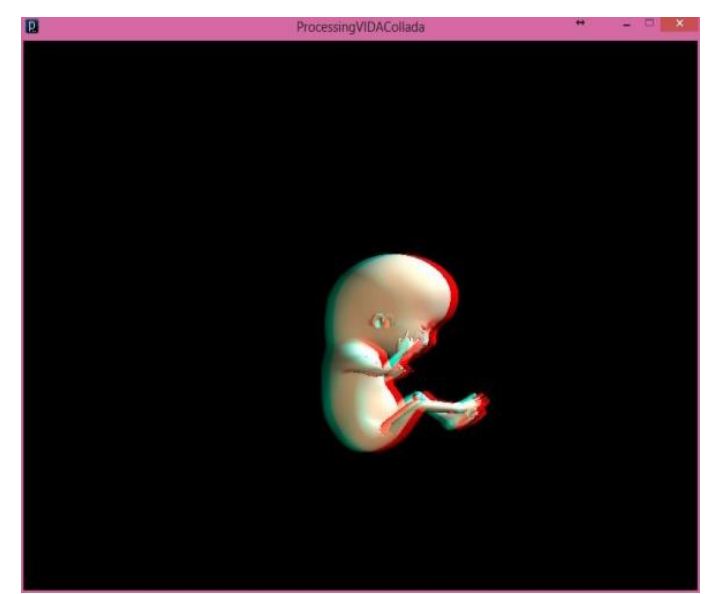

Figura 7. Movimento realizado no Projeto VIDA agregado ao SAAp

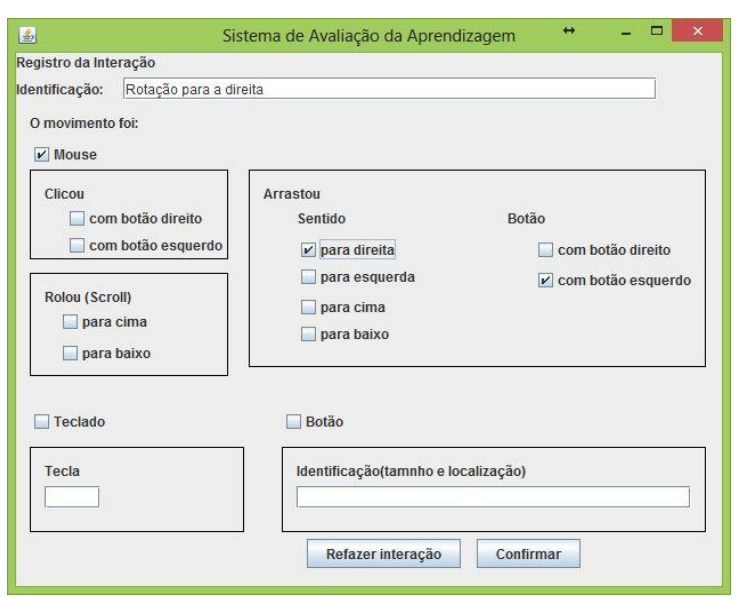

Figura 8. Registro da interação realizada no VIDA

Como segundo estudo de caso, foram realizados testes usando a ferramenta computacional voltada para o ensino de Geometria Plana e Espacial, denominada CONSTRUFIG3D [Mendes, Carvalho e Carvalho 2007]. Os autores desenvolveram a ferramenta para auxiliar alunos que estão iniciando o estudo de Geometria. O sistema auxilia na identificação de figuras geométricas planas e espaciais, e permite a composição de figuras espaciais a partir da seleção de figuras planas. Por meio de uma abordagem jogo/simulação o aluno pode desenvolver conceitos sobre figuras espaciais a partir de figuras planas.

O ambiente apresenta os seguintes componentes: i) botões para selecionar o número de figuras planas que compõem a figura espacial (área 2D), ii) figuras planas usadas para compor a figura espacial (área 2D), iii) botões diversos como limpar, 
rotacionar, sair e botão 3D que tem como finalidade criar a figura espacial a partir das informações enviadas (área 2D). Para utilizar o ambiente o usuário escolhe de forma livre figuras geométricas planas, que irão compor uma figura espacial. Se as figuras planas escolhidas forem coerentes, o usuário irá visualizar e interagir com a figura espacial gerada na área de trabalho 3D, na qual é possível interagir com a figura, como pode ser visto na Figura 9. O CONSTRUFIG3D também é integrado ao SAAp e o registro das interações é armazenado em tempo real durante a execução da interação no próprio ambiente, como ilustram as Figuras 9 e 10.

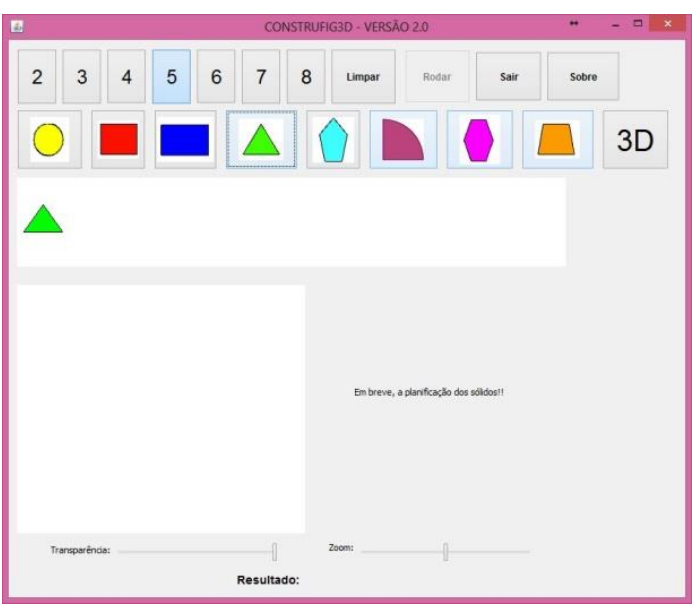

Figura 9. Evento - botão selecionado na interface CONSTRUFIG3D

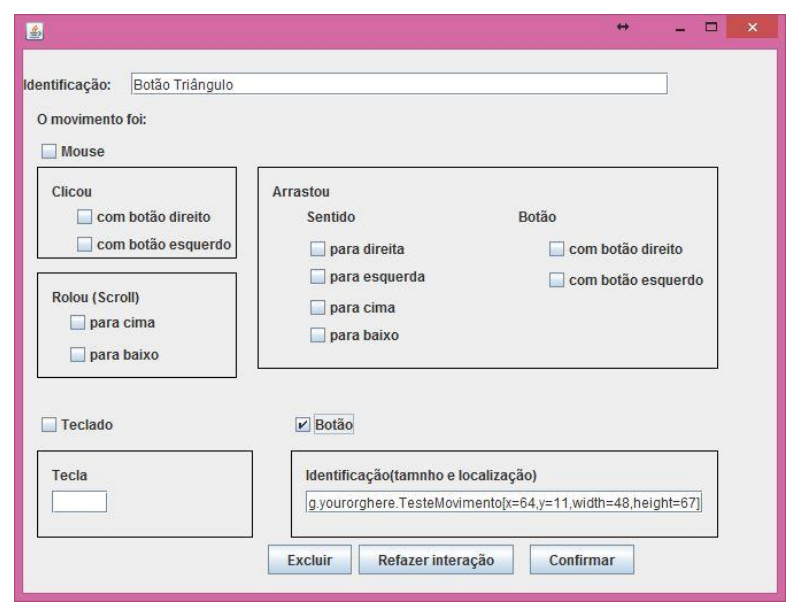

Figura 10. Registro da interação realizada no CONSTRUFIG3D

O CONSTRUFIG3D apresenta a área 3D para manipulação do objeto virtual, disponibilizando ações de rotação e escala, apresenta também uma carga expressiva de botões no componente da interface (área 2D), o que pode conduzir o usuário a uma maior frequência de interações com o ambiente, especialmente em relação às tentativas de criar diferentes figuras geométricas espaciais. Já o Projeto VIDA tem suas interações focadas na manipulação do ambiente usando os dispositivos mouse e teclado para interagir diretamente com o objeto virtual (feto), sendo possível realizar apenas interações do tipo manipulação como rotação, translação e escala, não apresentando nenhum outro componente na interface, a não ser o próprio objeto virtual.

Vale salientar que no perfil de participante o registro das interações também ocorre em tempo real, mas de forma invisível ao usuário, pois ele interage com o AVA 3D de forma livre, durante o tempo estipulado pelo avaliador, tendo as suas interações registradas automaticamente por meio do módulo de monitoramento das interações dos usuários implementado no Sistema de Avaliação da Aprendizagem.

A partir dos testes realizados, é possível afirmar que o módulo de monitoramento das interações do usuário foi capaz de interceptar todos os eventos previamente definidos, obtendo sucesso no registro das interações. Os resultados dos testes confirmam que o uso da POA foi adequado ao desenvolvimento desta pesquisa, devido as suas características, e por ser possível executar o módulo de registro das interações dos usuários em um maior número possível de AVAs 3D (plataforma Java). A solução adotada é válida para ambientes implementados na plataforma de programação Java, mas os conceitos podem ser expandidos para outras plataformas. Além dos resultados obtidos em relação ao monitoramento das interações, o Sistema de 
Avaliação da Aprendizagem se mostrou adequado, pois automatizou a coleta de dados por meio dos instrumentos de avaliação implementados.

Os dados coletados são exibidos ao avaliador por meio de relatórios gerados pelo SAAp, com possibilidade de serem exportados para uma planilha eletrônica. A Figura 11 ilustra um exemplo de relatório gerado pelo sistema, no qual exibe os dados referentes às interações e avaliações diagnósticas realizadas por um determinado participante.

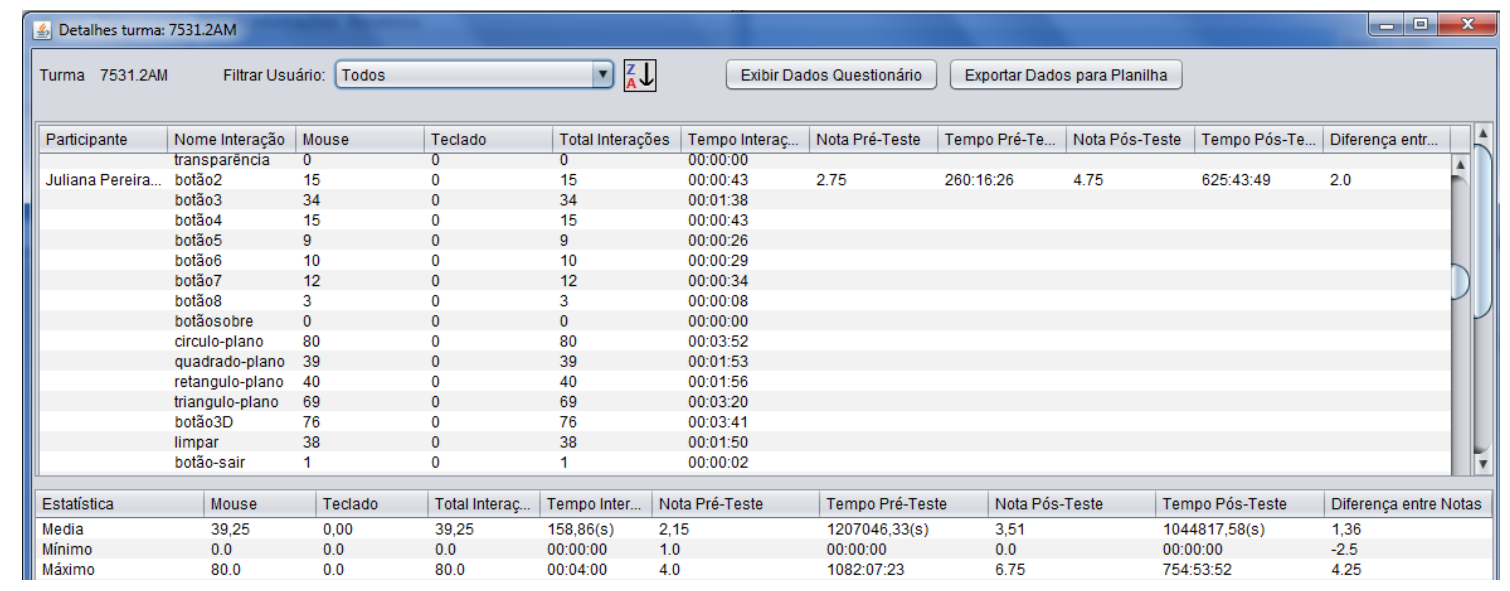

Figura 11. Exemplo de relatório gerado pelo SAAp

A partir de todos os dados coletados por meio do SAAp, inclusive o registro das interações durante a exploração no AVA 3D, é possível o avaliador analisar e verificar por meio de critérios e padrões, se de fato, tais ambientes estão contribuindo para a aquisição de conhecimento do aprendiz.

\section{Conclusões}

O Sistema de Avaliação da Aprendizagem apresentado neste trabalho se mostrou adequado para coletar os dados de aprendizagem por meio dos instrumentos de avaliação e monitoramento das interações dos participantes durante o processo de aprendizagem no AVA 3D.

O monitoramento das interações, que ocorre de forma automática, não intrusiva ao código fonte da aplicação é um objetivo difícil de ser alcançado completamente, visto que certas particularidades do código fonte podem confundir os métodos responsáveis pelo monitoramento. No entanto, verificou-se que nos AVAs 3D utilizados como estudo de caso, o módulo de monitoramento mostrou-se adequado.

Sendo assim, este estudo caracteriza uma contribuição importante à comunidade científica das áreas de Informática na Educação, Realidade Virtual e Aumentada, como também áreas correlatas que fazem uso de AVAs 3D e que tem interesse em coletar dados relacionados à aprendizagem, para posteriormente realizar análises/julgamentos acerca da evolução da aprendizagem por meio de AVAs 3D.

\section{Agradecimentos}

Os autores agradecem ao CNPq - Processo 559931/2010-7, ao Instituto Nacional de Ciência e Tecnologia de Medicina Assistida por Computação Científica (INCTMACC), à Fundação de Amparo à Pesquisa do Estado de São Paulo (Fapesp) - Processo 
2010/15691-0 e ao Programa Institucional de Bolsas de Iniciação Científica em Desenvolvimento Tecnológico e Inovação - PIBITI/CNPq/USP, pelo apoio financeiro.

\section{Referências}

AspectJ. The AspectJ Project. Disponível em $<$ http://www.eclipse.org/aspectj/>. Acessado em $28 / 03 / 2012$.

Camargo, V. V.; Masiero, P. C. (2006) Desenvolvimento de Software Orientado a Aspectos. In: Ildeberto Rodello, Kalinka Castelo Branco, José Remo. (Org.). II Escola Regional de Informática do Oeste Paulista. 1ed. Marília-SP, p. 138-164.

Mendes, J. L. S.; Carvalho, J. V.; Carvalho, C. V. A. (2007) CONSTRUFIG3D: Uma Ferramenta Computacional para apoio ao ensino da Geometria Plana e Espacial. RENOTE - Revista Novas Tecnologias na Educação 5.1.

Mikropoulos, T. A.; Natsis, A. (2011) Educational Virtual Environments: A ten-year review of empirical research (1999-2009). Computer Education, vol. 56, p. 769-780.

Nunes, E. P. S.; Nunes, F. L. S.; Tori, T.; Nunes, C. (2010) Definindo Parâmetros para Avaliação da Aquisição de Conhecimento em Ambientes Virtuais 3D. III Workshop sobre Avaliação e Acompanhamento da Aprendizagem em Ambientes Virtuais. João Pessoa, PB: XXI Simpósio Brasileiro de Informática na Educação - SBIE.

Nunes, E. P. S; Nunes, F. L. S.; Tori, R.; Kenski, V. M. (2012) A Model of assessment of knowledge acquisition in Three-Dimensional Learning Virtual Environments. In: XIV Symposium on Virtual and Augmented Reality, Niteroi. Proc. XIV Symposium on Virtual and Augmented Reality. Porto Alegre: SBC, 2012. p. 21-30.

Schlemmer, E.; Backes, L. (2008) Metaversos: novos espaços para construção do conhecimento. Revista Diálogo Educacional. Curitiba: Pontifícia Universidade Catolica do Paraná. 8, no. 24: pp. 519-532.

Sherman, W. R.; Craig, A. B. (2003) Understanding Virtual Reality: interface, application, and design. San Francisco, CA: Morgan Kaufmann Publishers.

Tori, R.; Nunes, F. L. S.; Gomes, V. H. P.; Tokunaga, D. M. (2009) VIDA: Atlas Anatômico 3D Interativo para Treinamento a Distância. In: WIE 2009 - XV Workshop Sobre Informática na Escola, 2009, Bento Gonçalves. Anais do XXIX Congresso da Sociedade Brasileira de Computação. Porto Alegre: SBC, pp. 1-10.

Valente, C.; Mattar, J. (2007) Second Life e WEB 2.0 na Educação: o potencial revolucionário das novas tecnologias. São Paulo: Novatec, ISBN 978-85-7522-147-1.

Vendruscolo, F.; Dias, J. A.; Bernardi, G.; Cassal, M.. (2005) Escola TRI-Legal - Um Ambiente Virtual como Ferramenta de Apoio ao Ensino Fundamental Através de Jogos Educacionais: Colabor@ - Revista Digital da CVA - RICESU. 3. 